\title{
Correlation of NRF2 and progesterone receptor and its effects on ovarian cancer biology
}

This article was published in the following Dove Press journal:

Cancer Management and Research

\author{
Bastian Czogalla' \\ Maja Kahaly' \\ Doris Mayr ${ }^{2}$ \\ Elisa Schmoeckel ${ }^{2}$ \\ Beate Niesler ${ }^{3}$ \\ Anna Hester' \\ Christine Zeder-Göß' \\ Thomas Kolben' \\ Alexander Burges \\ Sven Mahner' \\ Udo Jeschke' \\ Fabian Trillsch ${ }^{\prime}$ \\ 'Department of Obstetrics and \\ Gynecology, University Hospital, LMU \\ Munich, Munich, Germany; ${ }^{2}$ Faculty of \\ Medicine, Institute of Pathology, Lmu \\ Munich, Munich, Germany; ${ }^{3}$ Department \\ of Human Molecular Genetics, Institute \\ of Human Genetics, University of \\ Heidelberg, Heidelberg, Germany
}

Purpose: This study aimed to investigate the potential prognostic impact of nuclear factor erythroid 2-related factor 2 (NRF2) and progesterone receptor A (PRA)/progesterone receptor $\mathrm{B}(\mathrm{PRB})$ in ovarian cancer patients which might be the rationale for putative new treatment strategies.

Patients and methods: The presence of NRF2 and PRA/PRB was investigated in 156 ovarian cancer samples using immunohistochemistry (IHC). Staining of NRF2 and PRA/ PRB was rated using the semi-quantitative immunoreactive score (IR score, Remmele's score) and correlated to clinical and pathological data. NRF2 and PRA/PRB expression were compared with respect to the overall survival (OS).

Results: NRF2 staining was different in both, the cytoplasm and nucleus between the histological subtypes ( $p=0.001$ and $p=0.02$, respectively). There was a significant difference in the PRA expression comparing all histological subtypes $(p=0.02)$. Histological subtypes showed no significant differences in the PRB expression. A strong correlation of cytoplasmic NRF2 and PRA expression was detected $(\mathrm{cc}=0.247, p=0.003)$ as well as of cytoplasmic NRF2 and PRB expression ( $c c=0.25, p=0.003$ ), confirmed by immunofluorescence double staining. Cytoplasmic NRF2 expression was associated with a longer OS (median 50.6 vs 32.5 months; $p=0.1$ ) as it was seen for PRA expression (median 63.4 vs 33.1 months; $p=0.08$ ), although not statistically significant. In addition, high PRB expression (median 80.4 vs 32.5 months; $p=0.04$ ) and concurrent expression of cytoplasmic NRF2 and PRA were associated with a significantly longer OS (median 109.7 vs 30.6 months; $p=0.02$ ). The same relationship was also noted for NRF2 and PRB with improved OS for patients expressing both cytoplasmic NRF2 and PRB (median 153.5 vs 30.6 months; $p=0.009$ ). Silencing of $N F E 2 L 2$ induced higher mRNA expression of $P G R$ in the cancer cell line OVCAR3 $(p>0.05)$ confirming genetic interactions of NRF2 and PR.

Conclusion: In this study, the combination of cytoplasmic NRF2 and high PRA/PRB expression was demonstrated to be associated with improved overall survival in ovarian cancer patients. Further understanding of interactions within the NRF2/AKR1C1/PR pathway could open new additional therapeutic approaches.

Keywords: nuclear factor erythroid 2-related factor 2, progesterone receptor, ovarian cancer, immunohistochemistry

\section{Introduction}

Ovarian cancer is one of the five most frequent cancer deaths among women with a five-year survival rate of less than $45 \%{ }^{1,2}$ The non-specific symptoms combined with an insufficient screening method often lead to a diagnosis in advanced tumor stage with a consecutively impaired prognosis. Recommended therapeutic approaches include primary cytoreductive surgery and platinum-based chemotherapy with anti-angiogenic
Correspondence: Bastian Czogalla

University Hospital, LMU Munich,

Marchioninistr 15, Munich 81377, Germany

Tel +4989440074775

Fax +498944007205I

Email Bastian.Czogalla@med.uni-

muenchen.de 
agents or PARP inhibitors. Most reliable prognostic markers include volume of residual disease after initial debulking surgery, the International Federation of Gynecology and Obstetrics (FIGO) stage, ascites volume, patient age, and histological subtype..$^{3-6}$ Epithelial ovarian carcinomas (EOC) are classified as serous, mucinous, endometrioid, and clear-cell histology, being distinguished in terms of phenotype, molecular background, and etiology. ${ }^{7}$ Research to identify new molecular prognostic markers needs to take this heterogeneity of ovarian cancer into account. A better understanding of the differences between ovarian cancer subtypes appears crucial to enable new diagnostic and therapeutic approaches.

Current investigations attribute an important impact for the development of ovarian cancer to oxidative stress. ${ }^{8}$ The nuclear factor erythroid-2-related factor 2 (NRF2) is a wellknown regulator of antioxidant and cytoprotective genes mediating cellular coping of oxidative stress. Whereas NRF2 is ubiquitously expressed at low levels in all human organs, tight regulation of this major cellular defense mechanism is crucial to maintain cellular homeostasis. Different cancer entities and cell lines exhibit high constitutive levels of NRF2. ${ }^{9-13}$ Our research group recently demonstrated that cytoplasmic NRF2 expression in its inactive cytoplasmic form is associated with improved survival in ovarian cancer patients. ${ }^{14}$ Overexpression of NRF2 might protect cancer cells from the cytotoxic effects of anticancer therapies, resulting in resistance to chemo- and radiotherapy. ${ }^{15,16}$

Progesterone inhibits cell growth and metastasis in ovarian cancer cells and is considered as an established protective factor for the development of ovarian cancer as part of combined oral contraceptives. ${ }^{17-20}$ The detailed molecular background of this mechanism has not yet been fully understood. The progesterone receptor (PR), a member of the steroid hormone receptor superfamily, is expressed in two isoforms, the PRA and PRB differing in their molecular weight. Studies show an up to date functional unknown dominant expression of PRB in ovarian carcinomas. ${ }^{21-23}$ Progesterone receptor expression has been described to be associated with improved overall (OS) and progression-free survival (PFS) due to its putative anti-proliferative effect. ${ }^{24-27}$ To our knowledge, interactions between NRF2 and PR are not well understood so far, but warrant further investigation based on the results of our present data.

This study aimed to investigate the potential prognostic impact of NRF2 and PR in ovarian cancer patients which might be the rationale for putative new treatment strategies.

\section{Materials and methods}

\section{Patients and specimens}

Tissue samples of 156 patients who underwent surgery for EOC at the Department of Obstetrics and Gynecology, Ludwig-Maximillian's-University Munich from 1990 to 2002, were analyzed in this study. Clinical data were obtained from the patient's charts and follow-up data from the Munich Cancer Registry. All samples had been formalin-fixated and paraffin-embedded (FFPE). Patients with benign or borderline tumors were excluded and no patients had neoadjuvant chemotherapy. Specialized pathologists for EOC examined and classified the samples for tumor grading: low $(\mathrm{n}=38)$, high $(\mathrm{n}=117)$, and histological subtypes: serous $(n=110)$, endometrioid $(n=21)$, clear cell $(\mathrm{n}=12)$, mucinous $(\mathrm{n}=13)$. Staging was performed using TNM and FIGO (WHO) classification: I $(n=35)$, II $(\mathrm{n}=10$, ) III $(\mathrm{n}=103)$, IV $(\mathrm{n}=3)$. Data on primary tumor extension were available in 155 cases: $\mathrm{T} 1(\mathrm{n}=40), \mathrm{T} 2$ $(\mathrm{n}=18), \mathrm{T} 3(\mathrm{n}=93), \mathrm{T} 4(\mathrm{n}=4)$ as well as data on lymph node involvement in 95 cases N0 $(n=43), N 1(n=52)$. Data on distant metastasis were available in nine cases M0 $(\mathrm{n}=3), \mathrm{M} 1(\mathrm{n}=6)$.

\section{Ethical approval}

This study was approved by the Ethics Committee of the Ludwig-Maximilians-University, Munich, Germany (approval number 227-09). All tissue samples used for this study were obtained from material from the archives of the Department of Obstetrics and Gynecology, University Hospital, LMU Munich, Munich, Germany, initially used for pathological diagnostics. The diagnostic procedures were completed before the current study was performed. All patients' data were fully anonymized, and the study was performed according to the standards set in the Declaration of Helsinki 1975. The ethics committee approved this consent process. During the analysis, the observers were fully blinded for patients' data.

\section{Immunohistochemistry}

Immunohistochemistry was performed as previously described by our lab. ${ }^{28}$ For NRF2 staining, paraffinembedded and formalin-fixed EOC samples were incubated with Anti-NRF2 (Abcam, Cambridge, UK, rabbit, monoclonal, clone EP1808) at a final concentration of $5.93 \mu \mathrm{g} / \mathrm{mL}$ (1:100 dilution) for $1 \mathrm{hr}$ at room temperature. Afterward, slides were incubated with isotype-matching $\mathrm{MACH} 3$ Rabbit AP Polymer Detection (Biocare Medical, Pacheco, 
CA, USA, catalog-number M3R533). The Permanent AP Red Kit (Zytomed Systems GmbH, Berlin, Germany, catalog-number ZUC-001) was used as chromogen. Slides were then counterstained with Gill's hematoxylin (Vector Laboratories, Burlingame, CA, USA). System controls were included.

For the detection of PR, paraffin-fixed tissue sections were dewaxed with xylol for 15 mins, then dehydrated in ascending concentrations of alcohol (70-100\%). Afterward, they were exposed for epitope retrieval for $10 \mathrm{mins}$ in a pressure cooker using sodium citrate buffer ( $\mathrm{pH}$ 6.0) containing $0.1 \mathrm{M}$ citric acid and $0.1 \mathrm{M}$ sodium citrate in distilled water. After cooling, slides were washed in PBS twice. Endogenous peroxidase activity was quenched by dipping in 3\% hydrogen peroxide (Merck, Darmstadt, Germany) in methanol for 20 mins. Non-specific binding of the primary antibodies was blocked by incubating the sections with "diluted normal serum" (10 mL PBS containing $150 \mu \mathrm{L}$ horse serum; Vector Laboratories, CA) for 20 mins at room temperature. Then, slides were incubated with the primary antibodies (PRA: 1:250 dilutions, SigmaAldrich, St. Louis, MO, USA, rabbit, polyclonal, clone R04125; PRB: 1:50 dilutions, Novocastra Reagents, Wetzlar, Germany, mouse, monoclonal, clone SAN27) at room temperature for 60 mins. After washing with PBS, slides were incubated in diluted biotinylated anti-serum secondary antibody $(10 \mathrm{~mL}$ PBS containing $50 \mu$ horse serum, Vector Laboratories, CA) for 30 mins at room temperature. After incubation with the avidin-biotin-peroxidase complex (diluted in $10 \mathrm{~mL}$ PBS, Vector Laboratories, CA) for 30 mins and repeated PBS washing, visualization was conducted using substrate and chromagen 3,3'-diaminobenzidine (DAB; Dako, Glostrup, Denmark, catalog-number K3468) for $8-10$ mins. Slides were then counterstained with Mayer's acidic hematoxylin (Waldeck-Chroma, Münster, Germany, catalog-number 2E-038) and dehydrated in an ascending series of alcohol followed by xylol. System controls were included.

\section{Staining evaluation}

All EOC specimens were examined with a Leitz (Wetzlar, Germany) photomicroscope and specific NRF2 and PR immunohistochemically staining reaction was observed in the nuclei and cytoplasm of the cells. The intensity and distribution pattern of NRF2 and PR staining were rated using the semi-quantitative immunoreactive score (IR score, Remmele's score). To obtain the IR score result, the optional staining intensity $(0=$ no, $1=$ weak, $2=$ moderate, and $3=$ strong staining) and the percentage of positive-stained cells ( $0=$ no staining, $1=<10 \%$ of the cells, $2=11-50 \%$ of the cells, $3=51-80 \%$ of the cells, and $4 \leq 81 \%$ ) were multiplied. NRF2 staining was successfully performed in 145 (93\%) of 156 EOC tissue specimens. Cut-off points for the IR scores were selected for the cytoplasmic and nuclear NRF2 staining considering the distribution pattern of IR scores in the collective. Nuclear and cytoplasmic NRF2 staining were regarded as negative with an IR score $0-2$, as low with IRS $4-8$, and as high with IRS $>8$. PRA and PRB stainings were successfully performed in all $156(100 \%)$ EOC specimens. Cellular PRA and PRB stainings were considered as negative with an IR score 0 and as positive with IRS $>0$.

\section{Immunofluorescence staining}

Mouse anti-NRF2 IgGs were diluted at 1:200 with a diluting medium (Dako, Hamburg, Germany), while rabbit anti-PRB polyclonal IgGs were diluted at 1:200. After washing, slides were incubated with Cy2-/Cy3-labeled antibodies (Dianova, Hamburg, Germany) as fluorescent secondary antibodies for 30 mins at room temperature in darkness to avoid fluorescence quenching. Cy2-labeled secondary antibodies were used at a dilution of 1:100 and Cy3-labeled antibodies at a dilution of 1:500. Finally, the slides were embedded in mounting buffer containing 4',6-diamino-2-phenylindole (DAPI, Vectastain, Vector Laboratories) for blue staining of the nucleus after washing and drying. Confocal laser scanning microscope images were acquired with Zeiss LSM 880 with Airyscan model for high-resolution visualization and analyzed with ZEN blue software.

\section{Cell line}

The human serous ovarian cancer cell line OVCAR3 was purchased from the American Type Culture Collection (ATCC, Rockville, MD, USA). Cells were maintained in culture in RPMI 1640 medium (ThermoFisher Scientific, Waltham, MA, USA) supplemented with $10 \%$ FBS in a humified incubator at $37^{\circ} \mathrm{C}$ under $5 \% \mathrm{CO}_{2}$.

\section{PCR}

RNA isolation was performed using the RNeasy Mini Kit (Qiagen, Venlo, Netherlands) and $1 \mu \mathrm{g}$ RNA was converted into first-strand cDNA using the MMLV Reverse Transcriptase 1st-Strand cDNA Synthesis Kit (Epicentre, Madison, WI, USA) according to the instructions of the manufacturer. The basal mRNA expressions of NFE2L2 and PGR were quantified by qPCR applying FastStart Essential DNA Probes Master and gene-specific primers 
(Roche, Basel, Switzerland). For normalization of expressions the housekeeping genes $\beta$-Actin and $G A P D H$ were used as reference controls.

\section{si-RNA}

The specific siRNA for NFE2L2 (Silencer Select Predesigned and Custom Designed siRNA, Ambion, Carlsbad, CA, USA) was kindly provided by Beate Niesler (Department of Human Molecular Genetics, University of Heidelberg). Cells were transfected with siRNA using Lipofectamine RNAiMAX reagent (Invitrogen, Carlsbad, CA, USA) to silence the expression of NFE2L2 in the cell line. RNA Isolation and mRNA quantification by qPCR was repeated as outlined earlier. mRNA expression levels of NFE2L2 and PGR in NFE2L2 downregulated cells were compared with NFE2L2 containing cells.

\section{Statistical analysis}

Statistical analysis was performed using SPSS 25.0 (v25, IBM, Armonk, New York). Distribution of clinical pathological variables was evaluated with the Chi-Square test. Mann-Whitney $U$ test was used to compare IR scores of NRF2/PR between different clinical and pathological subgroups. Correlations between findings of immunohistochemically staining were calculated using Spearman's analysis. Survival times were analyzed by Kaplan-Meier (log-rank) estimates. To identify an appropriate cut-off, the ROC curve was drawn which is considered as one of the most reliable methods for cut-off point selection. In this context, the ROC curve is a plot representing sensitivity on the $y$-axis and (1-specificity) on the $x$-axis. ${ }^{29}$ Consecutively, Youden index, defined as the maximum (sensitivity+specificity-1), ${ }^{30}$ was used to find the optimal cut-off maximizing the sum of sensitivity and specificity. ${ }^{31,32}$ For multivariate analyses, a Cox-regression model was applied, with $P$-values less than 0.05 considered to be significant. $\mathrm{Ct}$ values of each gene were obtained with qPCR and the relative expressions were calculated using the $2^{-\Delta \Delta C t}$ formula. Statistical data were acquired using Graph Pad Prism 7.03 (v7, La Jolla, California).

\section{Results}

\section{NRF2/PR expression correlates with clinical and pathological data}

Clinicopathologic characteristics of the analyzed ovarian cancer patients are listed in Table 1. Nuclear staining of NRF2 was observed in 144 of 145 evaluable cases (99\%), and cytoplasmic staining of NRF2 was observed in 139
$(96 \%)$ of these 145 cases. Median (range) immunoreactivity scores (IRS) for NRF2 in nuclei and cytoplasm were 8 $(2,12)$ and $8(4,12)$, respectively.

NRF2 staining in both cytoplasm and nucleus was different between the histological subtypes $(p=0.001$ and $p=0.02$, respectively) with low nuclear NRF2 expression in serous, clear-cell, and endometrioid histology and high expression in mucinous subtype. In comparison, strongest and weakest cytoplasmic NRF2 staining were found in the serous and clear-cell subtypes, respectively. Cytoplasmic NRF2 expression was significantly higher expressed in patients with low-grade histology $(p=0.03)$ and low nuclear NRF2 expression was associated with age $(p=0.045)$.

All 156 cases could be successfully stained for PRA (100\%) and PRA expression could be detected in 63 of $156(40 \%)$ specimens with a median (range) IRS of 0 $(0,12)$ and mean (range) IRS of $2(0,12)$ (Figure 1$)$. There was a significant difference in the PRA expression comparing all histological subtypes $(p=0.02)$ with the highest expression in the serous subtype. All other

Table I Clinicopathologic characteristics of the ovarian cancer patients

\begin{tabular}{|l|l|l|}
\hline Clinicopathologic parameters & N & Percentage \\
\hline Histology & & \\
Serous & 110 & $70.5 \%$ \\
Clear cell & 12 & $7.7 \%$ \\
Endometrioid & 21 & $13.5 \%$ \\
Mucinous & 13 & $8.3 \%$ \\
\hline Lymph node & & \\
PN0/X & 104 & $66.7 \%$ \\
PNI & 52 & $33.3 \%$ \\
\hline Distant metastasis & & \\
PM0/X & 150 & $96.2 \%$ \\
PMI & 6 & $3.8 \%$ \\
\hline Grading & & \\
Low & 38 & $25.0 \%$ \\
High & 117 & $75.0 \%$ \\
\hline FIGO & & \\
I & 35 & $22.4 \%$ \\
II & 10 & $6.4 \%$ \\
III & 103 & $66.0 \%$ \\
IV & 3 & $1.9 \%$ \\
\hline Age & 73 & \\
S60 years & & $53.2 \%$ \\
$>60$ years & & $46.8 \%$ \\
\hline
\end{tabular}




\section{A NRF2}

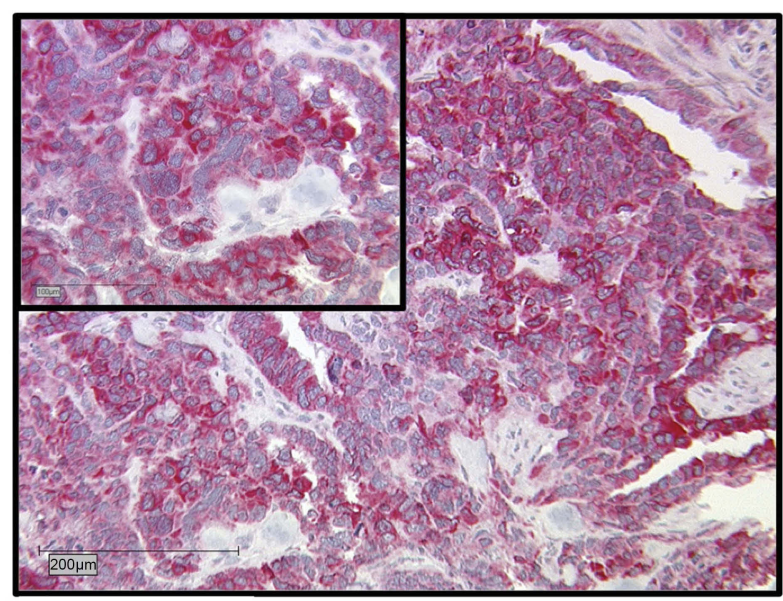

\section{B PRA}

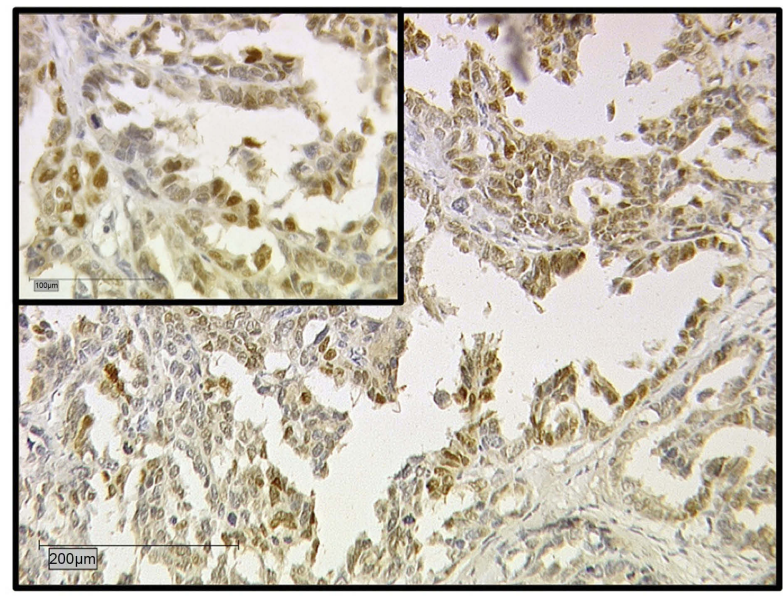

\section{PRB}

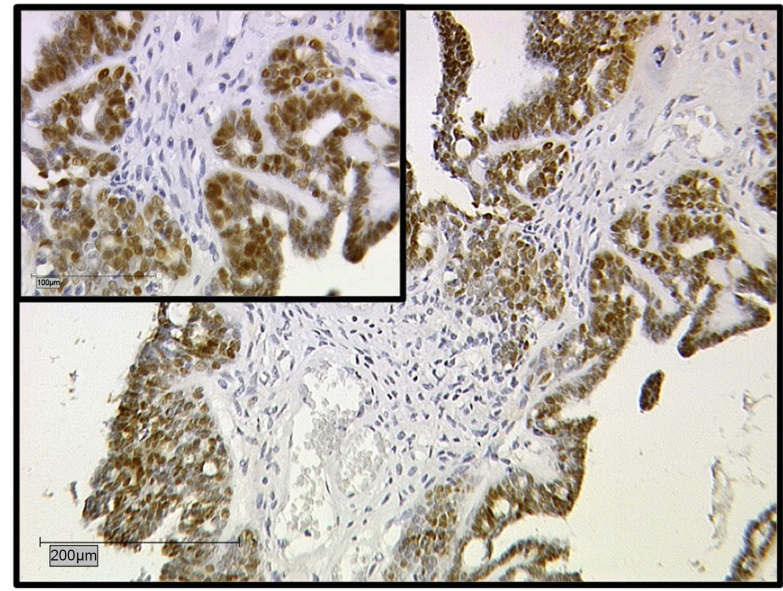

Figure I Detection of NRF2 and PRA/PRB with immunohistochemistry. Notes: High cytoplasmic NRF2 staining (A) corresponds with high PRA (B) and high PRB (C) staining found in specimens from the same individual. NRF2 cytoplasmic expression correlated with PRA ( $c c=0.247, p=0.003)$ and PRB expression $(c c=0.25, p=0.003)$. Abbreviations: $c c$, correlation coefficient, $p=$ two-tailed significance.

parameters like grading, FIGO, lymph node involvement $(\mathrm{pN})$, and distant metastasis ( $\mathrm{pM})$ showed no significant differences in the PRA expression. Of note, a strong correlation of cytoplasmic NRF2 and PRA expression was detected ( $\mathrm{cc}=0.247, p=0.003$, Table 2 and Figures 1 and 3).

PRB staining was successfully performed in all 156 cases $(100 \%)$ and PRB expression was observed in 63 of $156(40 \%)$ specimens with a median (range) IRS of 0 $(0,12)$ and mean (range) IRS of $2(0,12)$ (Figure 1). Parameters like histological subtypes, grading, FIGO, lymph node involvement $(\mathrm{pN})$, and distant metastasis (pM) showed no significant differences in the PRB expression. Again, NRF2 cytoplasmic expression was correlated with PRB expression ( $\mathrm{cc}=0.25, p=0.003$, Table 2 and Figures 1-3).

Table 2 Correlation analysis

\begin{tabular}{|c|l|l|l|}
\hline Staining & NRF2 cytoplasm & PRA & PRB \\
\hline NRF2 cytoplasm & & & \\
cc & 1.000 & 0.247 & 0.25 \\
$p$ &. & 0.003 & 0.003 \\
n & 146 & 142 & 144 \\
\hline PRA & & & \\
cc & 0.247 & 1.000 & 0.622 \\
p & 0.003 &. & 0.0001 \\
n & 142 & 152 & 152 \\
\hline PRB & & & \\
cc & 0.25 & 0.622 & 1.000 \\
P & 0.003 & $0.000 \mathrm{I}$ &. \\
n & 144 & 152 & 154 \\
\hline
\end{tabular}

Notes: IR-scores of NRF2 and PRA/PRB staining were correlated to each other using Spearman's correlation analysis.

Abbreviations: $c c$, correlation coefficient, $p$, two-tailed significance, n, number of patients.
NRF2
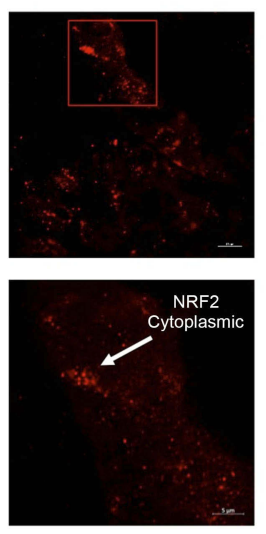

PRB
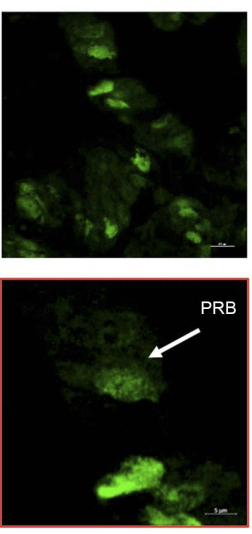

merge
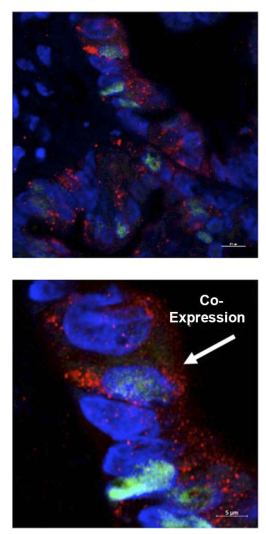

Figure 2 Double immunofluorescence of NRF2 and PRB.

Notes: Red stained cytoplasmic NRF2 expression, green stained PRB expression in ovarian cancer tissue. Co-expression of NRF2 and PRB + DAPI (triple filter excitation) 
A

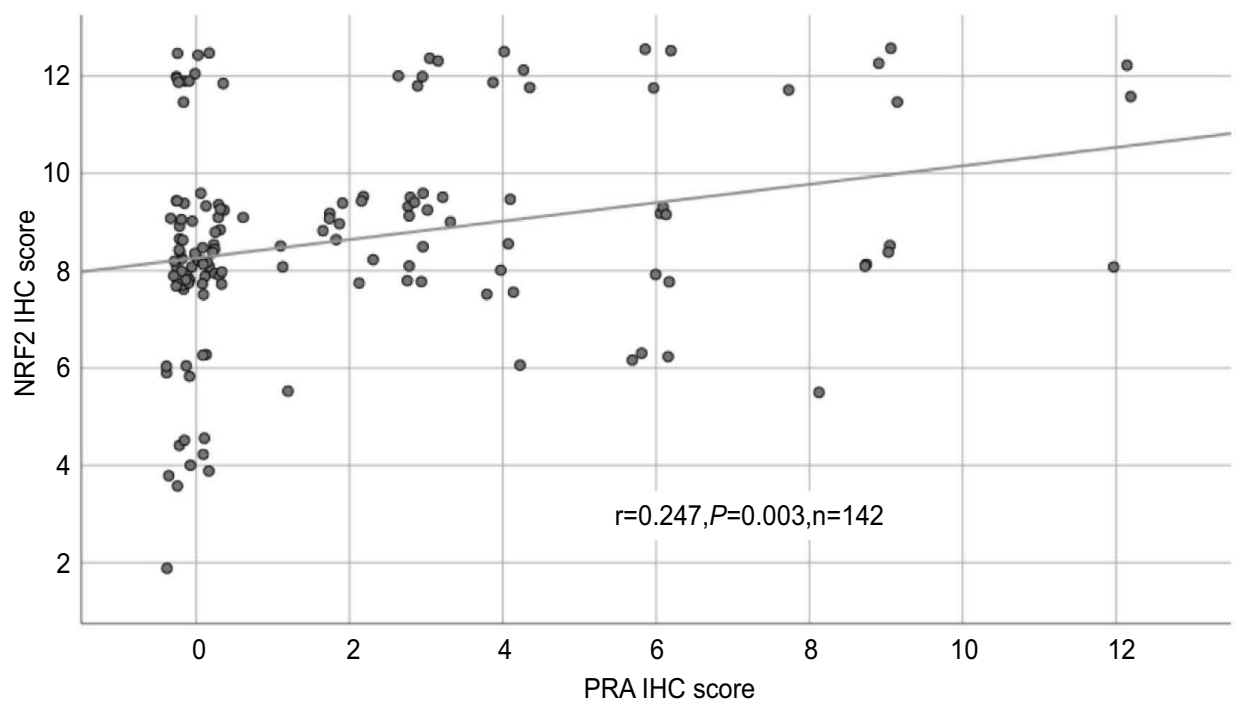

B

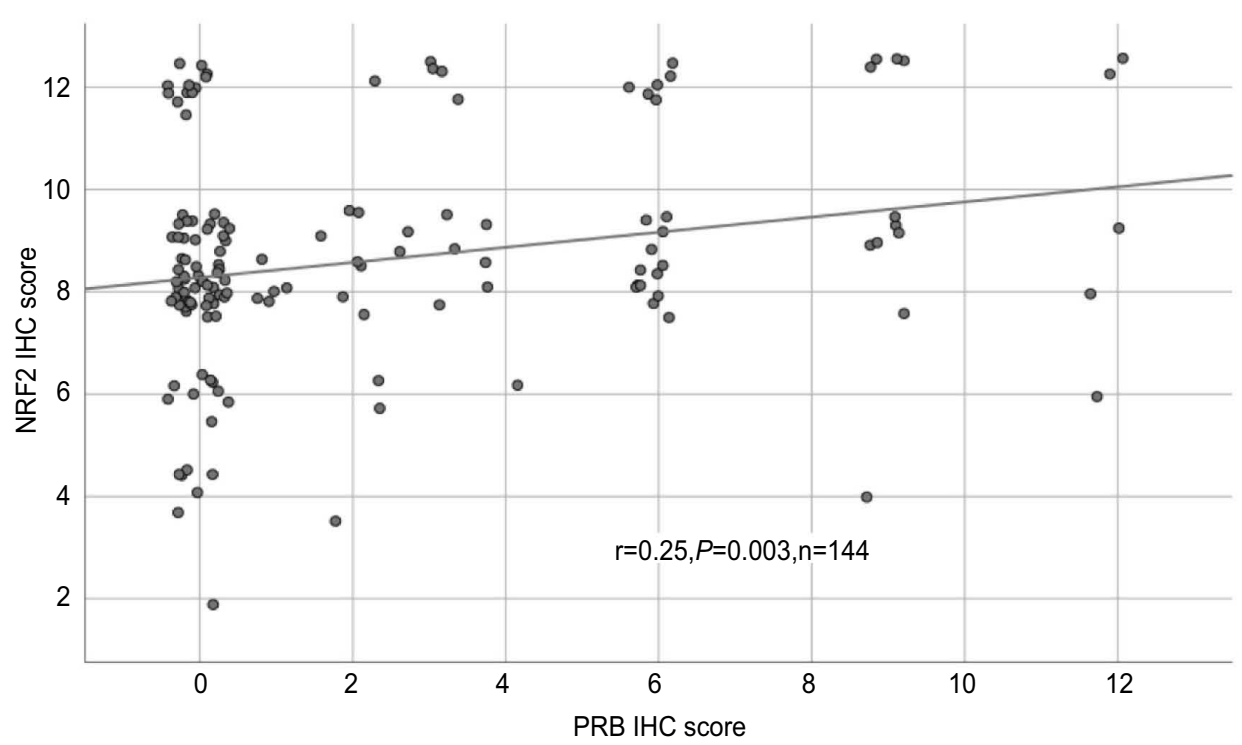

Figure 3 Correlation analysis of NRF2 and PRA/PRB.

Notes: Correlation analysis of NRF2 and PRA (A)/PRB (B) in ovarian cancer tissue. A significant correlation of cytoplasmic NRF2 expression with PRA/PRB expression was noted. For better visualization, dots have been jittered.

Abbreviations: $r=$ correlation coefficient, $p=$ two-tailed significance, $n=$ number of patients.

High NRF2/PR expression is associated with improved overall survival

Median age of the patients was 58.7 (standard deviation

[SD] 31.4) years with a range of 31-88 years. Median follow-up OS of the EOC patients was 34.4 (SD 57.8) months. Although not statistically significant, cytoplasmic NRF2 expression was associated with a longer OS (Figure 4 , median 50.6 vs 32.5 months; $p=0.1$ ) as it was seen for PRA expression (Figure 4, median 63.4 vs 33.1 months; $p=0.08)$. In addition, high PRB expression was associated with increased OS (Figure 4, median 80.4 vs 32.5 months; $p=0.04$ ).

Due to the biological relationship between NRF2 and PRA, concurrent expression of cytoplasmic NRF2 and PRA was evaluated revealing significantly longer OS for patients expressing both, NRF2 and PRA (Figure 4, median 109.7 vs 30.6 months; $p=0.02$ ). The same relationship was also noted between NRF2 and PRB with improved OS for patients with the combined expression of cytoplasmic 
A NRF2 cytoplasm

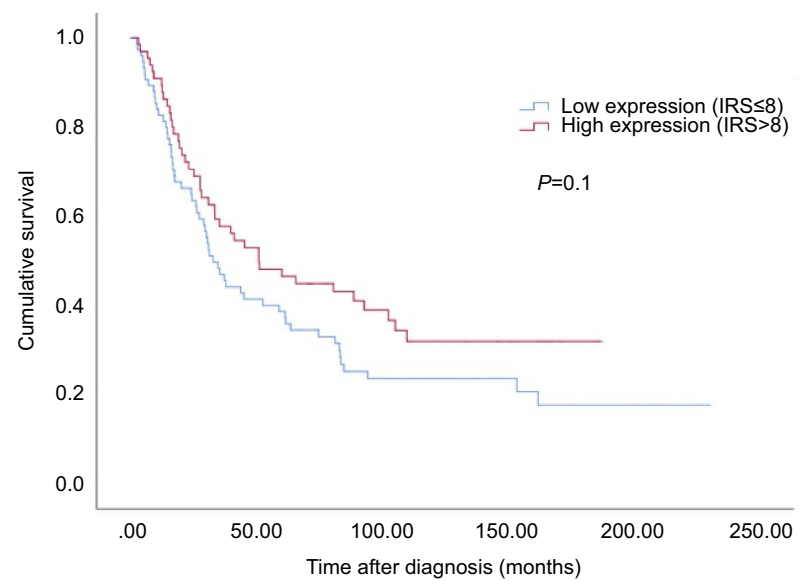

B PRA

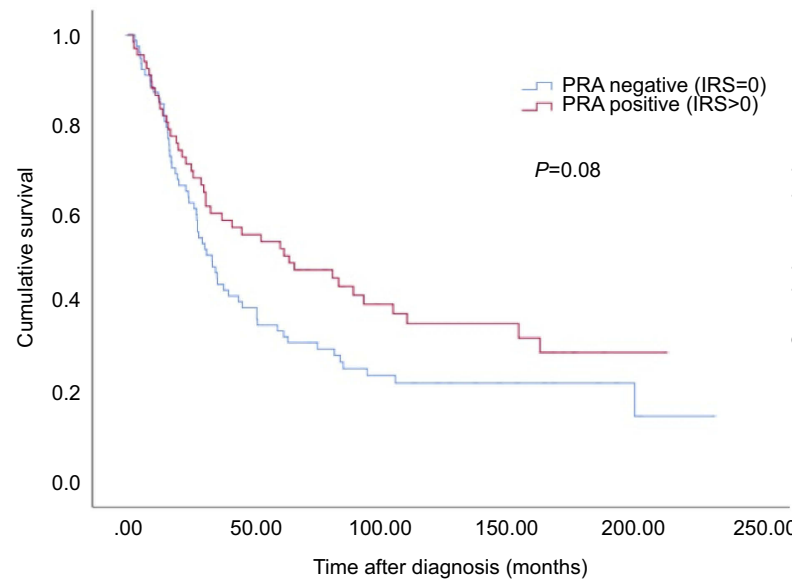

D NRF2 cytoplasm,PRB positive (IRS>0)

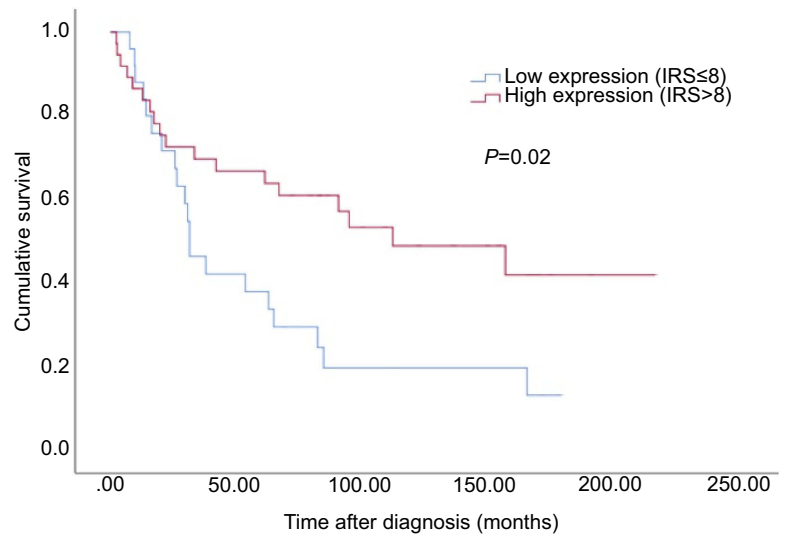

C PRB

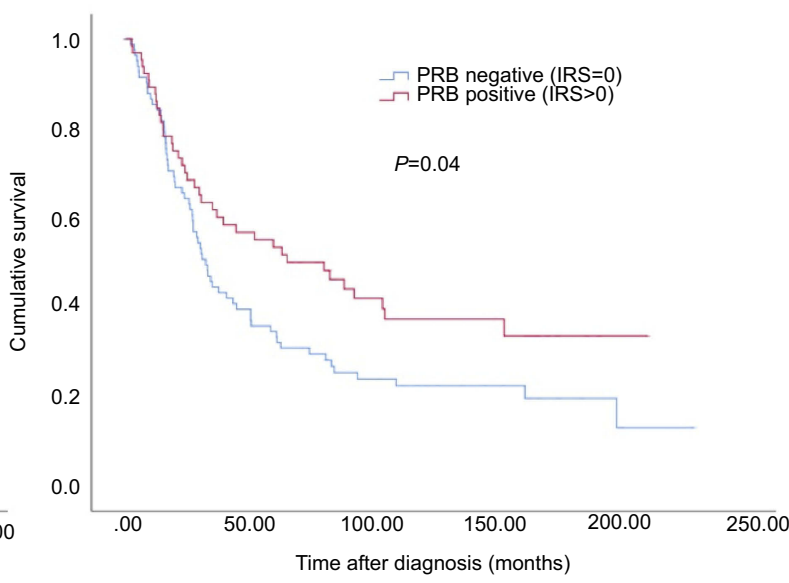

E

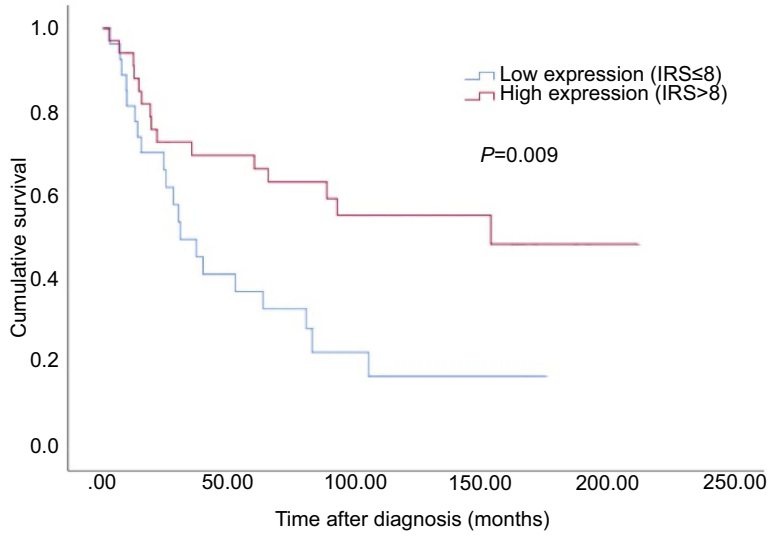

Figure 4 Kaplan-Meier estimates.

Notes: Kaplan-Meier estimates of NRF2 expression (A), PRA expression (B), PRB expression (C) and combined NRF2 and PRA/PRB (D, E) expression were analyzed. Although not statistically significant, cytoplasmic NRF2 expression was noted with a longer overall survival (A) as it was seen for PRA expression (B). High PRB expression was associated with increased overall survival (C). Patients with combined high NRF2 expression in the cytoplasm and PRA/PRB expression had significantly increased overall survival compared with those with low cytoplasmic expression (D/E). 
NRF2 and PRB (Figure 4, median 153.5 vs 30.6 months $p=0.009$ ).

\section{Clinical and pathological parameters are independent prognostic factors}

Cancer grading, the FIGO classification, and patient's age were independent prognostic factors in the present cohort (Table 3). In contrast, the prognostic impact of histological subtype as well as NRF2 and PRA/PRB expression were not confirmed to be of independent significance.

\section{Downregulation of NFE2L2 influences PGR expression confirming their genetic interaction}

Following effective silencing of NFE2L2 with siRNA to evaluate the impact on $P G R$ expression (Figure 5), an elevated expression of $P G R$ in the NFE2L2 downregulated cancer cell line OVCAR3 was noted, although not statistically significant $(p=0.41)$.

\section{Discussion}

The present study investigating the expression patterns of NRF2 and PRA as well as PRB demonstrates that cytoplasmic NRF2 expression is significantly correlated with the expression of both PRA and PRB and that this correlation seems to be associated with a significant impact on OS of ovarian cancer patients. Silencing of NFE2L2 induced a higher mRNA expression of $P G R$ in the NFE2L2 downregulated cancer cell line OVCAR3. Therefore, these results might corroborate a possible functional interaction between NRF2 and PR which merits further investigations.

As a main cellular defense mechanism against metabolic, xenobiotic, and oxidative stress, NRF2 has been generally regarded as a tumor suppressor. ${ }^{33,34}$ NRF2 activation avoids excessive cellular damage under abovementioned conditions. $^{34}$ Thus, NRF2/Keap1 pathway is essential in cancer chemoprevention underlining NRF2/ Keap1 mutations at pre-neoplastic stages in experimental models. ${ }^{35}$ In comparison, recent studies revealed that

Table 3 Multivariate analysis

\begin{tabular}{|c|c|c|c|c|c|}
\hline \multirow[t]{2}{*}{ Covariate } & \multirow[t]{2}{*}{ Coefficient $\left(\mathbf{b}_{\mathrm{i}}\right)$} & \multirow[t]{2}{*}[HR\operatorname{Exp}(b_{i})]{} & \multicolumn{2}{|l|}{$95 \% \mathrm{Cl}$} & \multirow[t]{2}{*}{$p$-Value } \\
\hline & & & Lower & Upper & \\
\hline Histology (serous vs other) & -0.124 & 0.883 & 0.678 & 1.188 & 0.35 \\
\hline Grade (low vs high) & 0.472 & 1.604 & 1.158 & 2.138 & 0.002 \\
\hline FIGO (I, II vs III, IV) & 0.679 & 1.972 & 1.550 & 3.096 & 0.000 \\
\hline Patients' age ( $\leq 60$ vs $>60$ years) & 0.008 & 1.008 & 1.003 & 1.013 & 0.001 \\
\hline NRF2 cytoplasmic/PRA & -0.090 & 0.914 & 0.382 & 2.332 & 0.85 \\
\hline NRF2 cytoplasmic/PRB & -0.422 & 0.656 & 0.276 & 1.639 & 0.35 \\
\hline
\end{tabular}

A

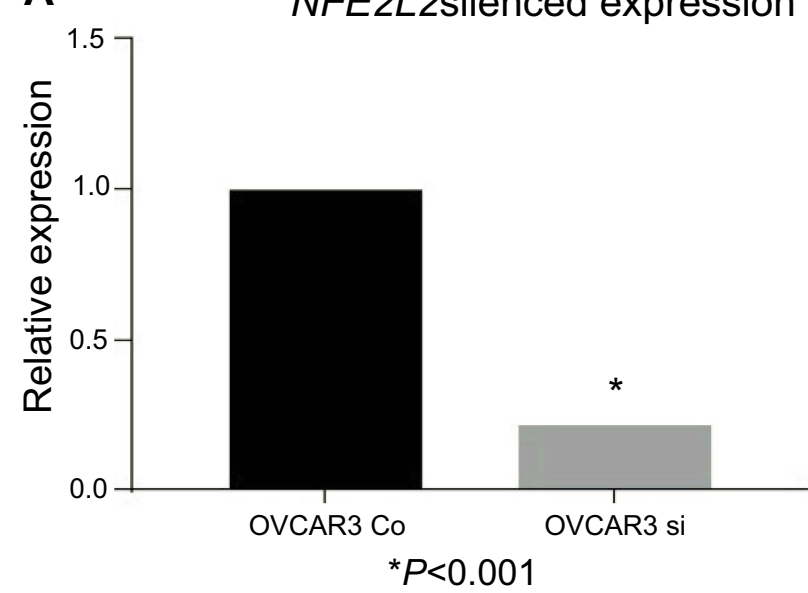

B

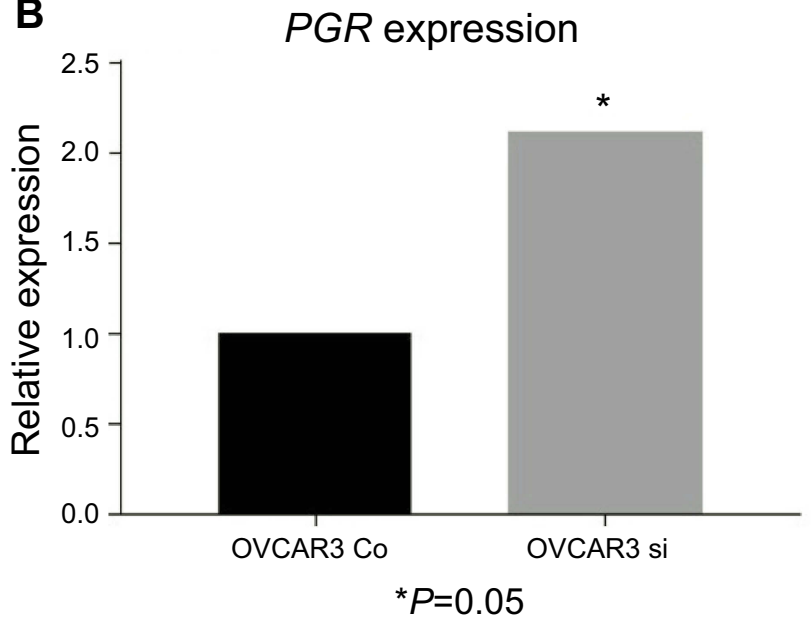

Figure 5 siRNA downregulation of NFE2L2.

Notes: siRNA downregulation of NFE2L2 in the ovarian cancer cell line OVCAR3 (A) and the effect on PGR expression following NFE2L2 downregulation (B). 
NRF2 hyperactivation may facilitate conditions favoring the survival of normal as well as malignant cells, protecting them from apoptosis following oxidative stress by chemotherapeutic agents or radiotherapy. ${ }^{36,37}$

Hence, this potential dual role of NRF2 in cancer biology should be taken into account in interpretation of its molecular role. Studies revealed that nuclear or activated NRF2 expression is associated with upregulation of multiple target genes with negative prognostic effects leading to impaired overall as well as progression-free survival (PFS). In accordance, patients with high cytoplasmic NRF2 expression (inactive form of the transcription factor) displayed improved OS and PFS. ${ }^{14,38}$

Progesterone plays an anti-proliferative effect via its receptor and has hereby been reported to be associated with improved OS and PFS in ovarian cancer patients. ${ }^{24-27}$ These findings are supported by studies showing that PR mediates apoptotic cell death. ${ }^{39,40}$ Furthermore, upregulation of Forkhead-box transcription factor (FOXO1) through progestin activated PR causes cell cycle arrest by increasing of mediators of cell senescence. ${ }^{40,41}$ A potential interaction between NRF2 and PR is not well understood yet, but can be conceived as a hypothesis-generating approach based on the presented evidence. NRF2 activates its target gene aldoketo reductase family 1 member $\mathrm{C} 1$ (AKR1C1) amongst others via an antioxidant response element (ARE) in a specific promoter region. AKR1C1 converts progesterone to its inactive form, the 20-alpha-dihydroxyprogesterone
(20-alpha-OHP). ${ }^{42,43}$ Moreover, AKR1C1 can bind to the promoter region of $\mathrm{PR}$ and decreases hereby receptor activity. $^{44}$ In this study, silencing of NFE2L2 induced a higher mRNA expression of $P G R$ supporting this data. In endometrial cancer patients, concurrent NRF2/AKR1C1 overexpression was proposed to be part of the molecular mechanisms underlying progestin resistance. ${ }^{45}$ Accordingly, increased expression of AKR1C1 is associated with the development of platinum resistance in human ovarian carcinoma cells as well as colon carcinoma cells. ${ }^{46,47}$ In contrast, progesterone facilitates the toxicity of cisplatin in ovarian cancer cells and a preclinical murine xenograft model. $^{48}$ Taken together, the interaction of NRF2 and PR might represent a potential pathway significantly influencing platinum response being mediated by AKR1C1 in ovarian cancer which should be followed in future studies. NRF2/ AKR1C1 expression can be downregulated by metformin treatment as described in endometrial and lung cancer cells. $^{45,49,50}$ Interestingly, recent studies show that metformin, usually applied in diabetic patients, prevents tumor growth, induces apoptosis and increases sensitivity to chemotherapy in ovarian cancer cells. ${ }^{51-57}$ Mechanisms underlying these cellular effects include suppression of cancer stem cells, inhibition of epithelial-to-mesenchymal transition and interference with neoplastic cell metabolism. ${ }^{58-62}$ Following promising data of epidemiological studies showing a favorable effect of metformin on ovarian cancer

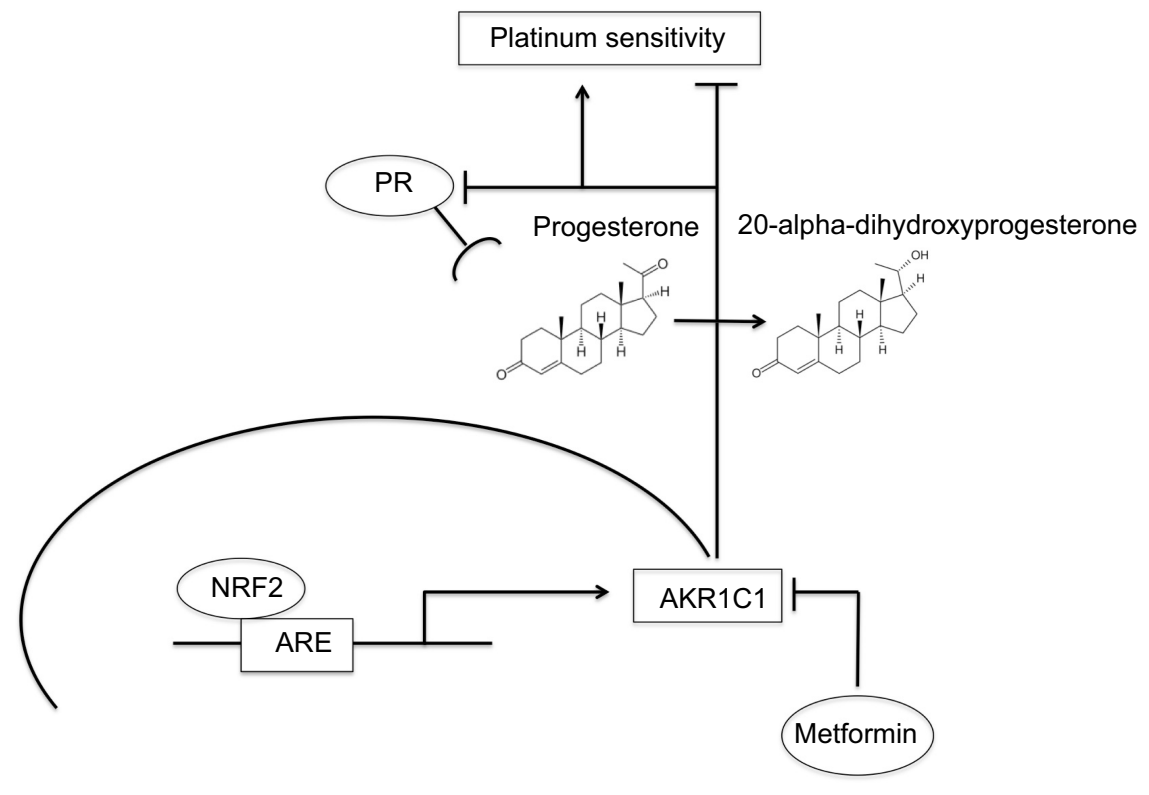

Figure 6 Summary of the hypothesized interaction within the NRF2/AKRICI/PR pathway.

Notes: Activated NRF2 (high nuclear, low cytoplasmic expression) activates aldo-keto reductase family I member $\mathrm{Cl}$ (AKRICI) via an antioxidant response element (ARE). $A K R I C l$ converts progesterone to its inactive form, the 20-alpha-dihydroxyprogesterone, and decreases PR receptor activity with consecutive platinum resistance. Metformin treatment counteracts this pathway, which may reverse the effects and consecutively lead to platinum re-sensitization. 
incidence and survival, it was proposed that metformin should be followed as an additional approach in ovarian cancer treatment. ${ }^{63-65}$ As metformin treatment is associated with an increased PR expression, these results further support future investigations of the above-described relationships in the NRF2/AKR1C1/PR pathway and their impact on ovarian cancer biology and the clinical behavior.

\section{Conclusion}

In summary, based on the results of the present study, we hypothesize that the interplay between NRF2/AKR1C1/PR might serve as an important pathway with significant impact on ovarian carcinogenesis elucidating additional therapeutic perspectives (Figure 6). With the rationale described earlier, metformin might have favorable effects on ovarian cancer biology and open new approaches to overcome platinum resistance which needs to be proved in future studies.

\section{Acknowledgments}

The authors are grateful to Mrs Martina Rahmeh and Mrs Christina Kuhn for excellent technical assistance. This work has been funded by the "Monika Kutzner" foundation and the "Brigitte \& Dr. Konstanze Wegener" foundation.

\section{Disclosure}

Bastian Czogalla has received a research grant from the "Monika Kutzner" foundation and "Brigitte \& Dr. Konstanze Wegener" foundation. Anna Hester has received a research grant from the "Walter Schulz" foundation and advisory board, speech honoraria and travel expenses from Roche and Pfizer. Thomas Kolben's relative is employed at Roche AG. Research support, advisory board, honoraria, and travel expenses from AstraZeneca, Clovis, Medac, MSD, Novartis, PharmaMar, Roche, Sensor Kinesis, Tesaro, Teva have been received by Sven Mahner and from AstraZeneca, Medac, PharmaMar, Roche, Tesaro by Fabian Trillsch. All authors report no other conflicts of interest in this work.

\section{References}

1. Siegel RL, Miller KD, Jemal A. Cancer statistics, 2017. CA Cancer J Clin. 2017;67(1):7-30. doi:10.3322/caac.21387

2. Baldwin LA, Huang B, Miller RW, et al. Ten-year relative survival for epithelial ovarian cancer. Obstet Gynecol. 2012;120(3). doi:10.1097/ AOG.0b013e318264f794

3. Du Bois A, Reuss A, Pujade-Lauraine E, Harter P, Ray-Coquard I, Pfisterer J. Role of surgical outcome as prognostic factor in advanced epithelial ovarian cancer: a combined exploratory analysis of 3 prospectively randomized phase 3 multicenter trials. Cancer. 2009;115 (6):1234-1244. doi:10.1002/cncr.24149
4. Aletti GD, Gostout BS, Podratz KC, Cliby WA. Ovarian cancer surgical resectability: relative impact of disease, patient status, and surgeon. Gynecol Oncol. 2006;100(1):33-37. doi:10.1016/j. ygyno.2005.07.123

5. Vergote I, De Brabanter J, Fyles A, et al. Prognostic importance of degree of differentiation and cyst rupture in stage I invasive epithelial ovarian carcinoma. Lancet. 2001;357(9251):176-182. doi:10.1016/ S0140-6736(00)03590-X

6. Dembo AJ, Davy M, Stenwig AE, Berle EJ, Bush RS, Kjorstad K. Prognostic factors in patients with stage I epithelial ovarian cancer. Obstet Gynecol. 1990;75(2):263-273. Available from: http://eur opepmc.org/abstract/MED/2300355. Accessed July 15, 2019.

7. Kossaï M, Leary A, Scoazec J-Y, Genestie C. Ovarian cancer: a heterogeneous disease. Pathobiology. 2018;85(1-2):41-49. doi:10.1159/ 000479006

8. van der Wijst MGP, Brown R, Rots MG. Nrf2, the master redox switch: the Achilles' heel of ovarian cancer? Biochim Biophys Acta. 2014;1846(2):494-509. doi:10.1016/j.bbcan.2014.09.004

9. Namani A, Matiur Rahaman M, Chen M, Tang X. Gene-expression signature regulated by the KEAP1-NRF2-CUL3 axis is associated with a poor prognosis in head and neck squamous cell cancer. $B M C$ Cancer. 2018;18(1):46. doi:10.1186/s12885-017-3907-z

10. Boustani MR, Khoshnood RJ, Nikpasand F, et al. Overexpression of ubiquitin-specific protease 2a (USP2a) and nuclear factor erythroid 2related factor 2 (Nrf2) in human gliomas. J Neurol Sci. 2016;363:249-252. doi:10.1016/j.jns.2016.03.003

11. Ji L, Wei Y, Jiang T, Wang S. Correlation of Nrf2, NQO1, MRP1, cmyc and p53 in colorectal cancer and their relationships to clinicopathologic features and survival. Int J Clin Exp Pathol. 2014;7 (3):1124-1131. Available from: https://www.ncbi.nlm.nih.gov/ pubmed/24695690. Accessed July 15, 2019.

12. Jiang $\mathrm{T}$, Chen N, Zhao F, et al. High levels of Nrf2 determine chemoresistance in type II endometrial cancer. Cancer Res. 2010;70 (13):5486-5496. doi:10.1158/0008-5472.CAN-10-0713

13. Ryoo I, Choi B, Kwak M-K. Activation of NRF2 by p62 and proteasome reduction in sphere-forming breast carcinoma cells. Oncotarget; . 2015;6(10). doi:10.18632/oncotarget.v6i10

14. Czogalla B, Kahaly M, Mayr D, et al. Interaction of ER $\alpha$ and NRF2 impacts survival in ovarian cancer patients. Int J Mol Sci. 2018;20:1. doi:10.3390/ijms20010112

15. Zhang DD. The Nrf2-Keap1-ARE signaling pathway: the regulation and dual function of Nrf2 in cancer. Antioxid Redox Signal. 2010;13 (11):1623-1626. doi:10.1089/ars.2010.3301

16. Lo R, Matthews J. The aryl hydrocarbon receptor and estrogen receptor alpha differentially modulate nuclear factor erythroid-2-related factor 2 transactivation in MCF-7 breast cancer cells. Toxicol Appl Pharmacol. 2013;270(2):139-148. doi:10.1016/j.taap.2013.03.029

17. Wu N-Y, Huang H-S, Chao TH, et al. Progesterone prevents highgrade serous ovarian cancer by inducing necroptosis of $\mathrm{p} 53$-defective fallopian tube epithelial cells. Cell Rep. 2017;18(11):2557-2565. doi:10.1016/j.celrep.2017.02.049

18. Jeon S-Y, Hwang K-A, Choi K-C. Effect of steroid hormones, estrogen and progesterone, on epithelial mesenchymal transition in ovarian cancer development. J Steroid Biochem Mol Biol. 2016;158:1-8. doi:10.1016/j.jsbmb.2016.02.005

19. Nagendra PB, Goad J, Nielsen S, et al. Ovarian hormones through Wnt signalling regulate the growth of human and mouse ovarian cancer initiating lesions. Oncotarget. 2016;7(40):64836-64853. doi:10.18632/oncotarget.11711

20. Lurie G, Wilkens LR, Thompson PJ, et al. Combined oral contraceptive use and epithelial ovarian cancer risk: time-related effects. Epidemiology. 2008;19(2). doi:10.1097/EDE.0b013e31816334c5

21. Akahira J, Inoue T, Suzuki T, et al. Progesterone receptor isoforms A and $\mathrm{B}$ in human epithelial ovarian carcinoma: immunohistochemical and RT-PCR studies. $B r \quad J$ Cancer. 2000;83(11):1488-1494. doi:10.1054/bjoc.2000.1463 
22. Akahira J-I, Suzuki T, Ito K, et al. Differential expression of progesterone receptor isoforms $\mathrm{A}$ and $\mathrm{B}$ in the normal ovary, and in benign, borderline, and malignant ovarian tumors. Jpn J Cancer Res. 2002;93 (7):807-815. doi:10.1111/j.1349-7006.2002.tb01323.x

23. Lenhard M, Tereza L, Heublein S, et al. Steroid hormone receptor expression in ovarian cancer: progesterone receptor $\mathrm{B}$ as prognostic marker for patient survival. BMC Cancer. 2012;12:553. doi:10.1186/ 1471-2407-12-553

24. Luo H, Li S, Zhao M, Sheng B, Zhu H, Zhu X. Prognostic value of progesterone receptor expression in ovarian cancer: a meta-analysis. Oncotarget. 2017;8(22):36845-36856. doi:10.18632/oncotarget.15982

25. Sinn BV, Darb-Esfahani S, Wirtz RM, et al. Evaluation of a hormone receptor-positive ovarian carcinoma subtype with a favourable prognosis by determination of progesterone receptor and oestrogen receptor 1 mRNA expression in formalin-fixed paraffin-embedded tissue. Histopathology. 2011;59(5):918-927. doi:10.1111/j.1365-2559.2011.04028.x

26. Sieh W, Köbel M, Longacre TA, et al. Hormone-receptor expression and ovarian cancer survival: an Ovarian Tumor Tissue Analysis consortium study. Lancet Oncol. 2013;14(9):853-862. doi:10.1016/ S1470-2045(13)70253-5

27. Diep CH, Daniel AR, Mauro LJ, Knutson TP, Lange CA. Progesterone action in breast, uterine, and ovarian cancers. $J \mathrm{Mol}$ Endocrinol. 2015;54(2):R31-R53. doi:10.1530/JME-14-0252

28. Scholz C, Heublein S, Lenhard M, Friese K, Mayr D, Jeschke U. Glycodelin A is a prognostic marker to predict poor outcome in advanced stage ovarian cancer patients. BMC Res Notes. 2012;5:551. doi:10.1186/1756-0500-5-551

29. Nakas CT, Alonzo TA, Yiannoutsos CT. Accuracy and cut-off point selection in three-class classification problems using a generalization of the Youden index. Stat Med. 2010;29(28):2946-2955. doi:10.1002/ $\operatorname{sim} .4044$

30. Youden WJ. Index for rating diagnostic tests. Cancer. 1950;3(1):3235. doi:10.1002/1097-0142(1950)3:1<32::AID-CNCR2820030106>3.0. $\mathrm{CO} ; 2-3$

31. Perkins NJ, Schisterman EF. The inconsistency of "optimal" cutpoints obtained using two criteria based on the receiver operating characteristic curve. Am J Epidemiol. 2006;163(7):670-675. doi:10.1093/aje/kwj063

32. Fluss R, Faraggi D, Reiser B. Estimation of the youden index and its associated cutoff point. Biometrical J. 2005;47(4):458-472. doi:10.1002/bimj.200410135

33. Villeneuve NF, Lau A, Zhang DD. Regulation of the Nrf2-keap1 antioxidant response by the ubiquitin proteasome system: an insight into cullin-ring ubiquitin ligases. Antioxid Redox Signal. 2010;13 (11):1699-1712. doi:10.1089/ars.2010.3211

34. Wakabayashi N, Slocum SL, Skoko JJ, Shin S, Kensler TW. When NRF2 talks, who's listening? Antioxid Redox Signal. 2010;13 (11):1649-1663. doi:10.1089/ars.2010.3216

35. Hayes JD, McMahon M, Chowdhry S, Dinkova-Kostova AT. Cancer chemoprevention mechanisms mediated through the Keap1-nrf2 pathway. Antioxid Redox Signal. 2010;13(11):1713-1748. doi:10.1089/ars.2010.3221

36. Menegon S, Columbano A, Giordano S. The dual roles of NRF2 in cancer. Trends Mol Med. 2016;22(7):578-593. doi:10.1016/j. molmed.2016.05.002

37. Taguchi K, Yamamoto M. The KEAP1-NRF2 system in cancer. Front Oncol. 2017;7:85. doi:10.3389/fonc.2017.00085

38. Cho H, Kim K, Kim Y-B, Kim H, No JH. Expression patterns of Nrf2 and Keap1 in ovarian cancer cells and their prognostic role in disease recurrence and patient survival. Int J Gynecol Cancer. 2017;27(3). doi:10.1097/IGC.0000000000000908

39. Modugno F, Laskey R, Smith AL, Andersen CL, Haluska P, Oesterreich S. Hormone response in ovarian cancer: time to reconsider as a clinical target? Endocr Relat Cancer. 2012;19(6):R255R279. doi:10.1530/ERC-12-0175
40. Chuffa LGDA, Lupi-Júnior LA, Costa AB, Amorim JPDA, Seiva FRF. The role of sex hormones and steroid receptors on female reproductive cancers. Steroids. 2017;118:93-108. doi:10.1016/j. steroids.2016.12.011

41. Diep C, Charles N, Blake Gilks C, Kalloger S, Argenta P, Lange CA. Progesterone receptors induce FOXO1-dependent senescence in ovarian cancer cells. Cell Cycle. 2013;12(9):1433-1449. doi: $10.4161 /$ cc. 24550

42. Nishizawa M, Nakajima T, Yasuda K, et al. Close kinship of human $20 \alpha$-hydroxysteroid dehydrogenase gene with three aldo-keto reductase genes. Genes Cells. 2000;5(2):111-125. doi:10.1046/j.13652443.2000.00310.x

43. Rižner TL, Šmuc T, Rupreht R, Šinkovec J, Penning TM. AKR1C1 and AKR1C3 may determine progesterone and estrogen ratios in endometrial cancer. Mol Cell Endocrinol. 2006;248(1):126-135. doi:10.1016/j.mce.2005.10.009

44. Ji Q, Aoyama C, Nien Y-D, et al. Selective loss of AKR1C1 and AKR1C2 in breast cancer and their potential effect on progesterone signaling. Cancer Res. 2004;64(20):7610 LP-7617. doi:10.1158/ 0008-5472.CAN-04-1608

45. Wang Y, Wang Y, Zhang Z, et al. Mechanism of progestin resistance in endometrial precancer/cancer through Nrf2-AKR1C1 pathway. Oncotarget. 2016;7(9):10363-10372. doi:10.18632/oncotarget.7004

46. Deng HB, Parekh HK, Chow K-C, Simpkins H. Increased expression of dihydrodiol dehydrogenase induces resistance to cisplatin in human ovarian carcinoma cells. J Biol Chem. 2002;277(17):1503515043. doi:10.1074/jbc.M112028200

47. Matsunaga T, Hojo A, Yamane Y, Endo S, El-Kabbani O, Hara A. Pathophysiological roles of aldo-keto reductases (AKR1C1 and AKR1C3) in development of cisplatin resistance in human colon cancers. Chem Biol Interact. 2013;202(1):234-242. doi:10.1016/j. cbi.2012.09.024

48. Murdoch WJ, Van Kirk EA, Isaak DD, Shen Y. Progesterone facilitates cisplatin toxicity in epithelial ovarian cancer cells and xenografts. Gynecol Oncol. 2008;110(2):251-255. doi:10.1016/j.ygyno.2008.03.021

49. Yu C, Jiao Y, Xue J, et al. Metformin sensitizes non-small cell lung cancer cells to an epigallocatechin-3-Gallate (EGCG) treatment by suppressing the Nrf2/HO-1 signaling pathway. Int J Biol Sci. 2017;13 (12):1560-1569. doi:10.7150/ijbs. 18830

50. Zhang J, Jiao K, Liu J, Xia Y. Metformin reverses the resistance mechanism of lung adenocarcinoma cells that knocks down the Nrf2 gene. Oncol Lett. 2018;16(5):6071-6080. doi:10.3892/ol.2018.9382

51. Lengyel E, Litchfield LM, Mitra AK, et al. Metformin inhibits ovarian cancer growth and increases sensitivity to paclitaxel in mouse models. Am J Obstet Gynecol. 2015;212(4):479.e1-479.e10. doi:10.1016/j.ajog.2014.10.026

52. Zheng Y, Zhu J, Zhang H, Liu Y, Sun H. Metformin inhibits ovarian cancer growth and migration in vitro and in vivo by enhancing cisplatin cytotoxicity. Am J Transl Res. 2018;10(10):3086-3098. Available from: https:// www.ncbi.nlm.nih.gov/pubmed/30416652. Accessed July 15, 2019.

53. Dos Santos Guimarães I, Ladislau-Magescky T, Tessarollo NG, et al. Chemosensitizing effects of metformin on cisplatin- and paclitaxelresistant ovarian cancer cell lines. Pharmacol Rep. 2018;70(3):409417. doi:10.1016/j.pharep.2017.11.007

54. Bishnu A, Sakpal A, Ghosh N, Choudhury P, Chaudhury K, Ray P. Long term treatment of metformin impedes development of chemoresistance by regulating cancer stem cell differentiation through taurine generation in ovarian cancer cells. Int $J$ Biochem Cell Biol. 2019;107:116-127. doi:10.1016/j.biocel.2018.12.016

55. Garrido MP, Vera C, Vega M, Quest AFG, Romero C. Metformin prevents nerve growth factor-dependent proliferative and proangiogenic effects in epithelial ovarian cancer cells and endothelial cells. Ther Adv Med Oncol. 2018;10:1758835918770984. doi:10.1177/1758835918770984

56. Tang G, Guo J, Zhu Y, et al. Metformin inhibits ovarian cancer via decreasing H3K27 trimethylation. Int J Oncol. 2018;52(6):18991911. doi:10.3892/ijo.2018.4343 
57. Dang J-H, Jin Z-J, Liu X-J, et al. Metformin in combination with cisplatin inhibits cell viability and induces apoptosis of human ovarian cancer cells by inactivating ERK 1/2. Oncol Lett. 2017;14 (6):7557-7564. doi:10.3892/ol.2017.7176

58. Vazquez-Martin A, López-Bonetc E, Cufí S, et al. Repositioning chloroquine and metformin to eliminate cancer stem cell traits in pre-malignant lesions. Drug Resist Updat. 2011;14(4-5):212-223. doi:10.1016/j.drup.2011.04.003

59. Cufi S, Vazquez-Martin A, Oliveras-Ferraros C, Martin-Castillo B, Joven J, Menendez JA. Metformin against TGF $\beta$-induced epithelial-to-mesenchymal transition (EMT): from cancer stem cells to aging-associated fibrosis. Cell Cycle. 2010;9(22):4461-4468. doi:10.4161/cc.9.22.14048

60. Shackelford DB, Shaw RJ. The LKB1-AMPK pathway: metabolism and growth control in tumour suppression. Nat Rev Cancer. 2009;9 (8):563-575. doi:10.1038/nrc2676

61. Zakikhani M, Dowling R, Fantus IG, Sonenberg N, Pollak M. Metformin is an AMP Kinase-dependent growth inhibitor for breast cancer cells. Cancer Res. 2006;66(21):10269 LP-10273 LP. doi:10.1158/0008-5472.CAN-06-1500
62. Lettieri Barbato D, Vegliante R, Desideri E, Ciriolo MR. Managing lipid metabolism in proliferating cells: new perspective for metformin usage in cancer therapy. Biochim Biophys Acta Rev Cancer. 2014;1845(2):317-324. doi:10.1016/j.bbcan.2014. 02.003

63. Shi J, Liu B, Wang H, Zhang T, Yang L. Association of metformin use with ovarian cancer incidence and prognosis: a systematic review and meta-analysis. Int J Gynecol Cancer. 2019;29(1):140 LP-146 LP. doi:10.1136/ijgc-2018-000060

64. Wang S-B, Lei K-J, Liu J-P, Jia Y-M. Continuous use of metformin can improve survival in type 2 diabetic patients with ovarian cancer: a retrospective study. Medicine (Baltimore). 2017;96(29):e7605e7605. doi:10.1097/MD.0000000000007605

65. Garcia C, Yao A, Camacho F, Balkrishnan R, Cantrell LA. A SEERmedicare analysis of the impact of metformin on overall survival in ovarian cancer. Gynecol Oncol. 2017;146(2):346-350. doi:10.1016/j. ygyno.2017.05.006

\section{Publish your work in this journal}

Cancer Management and Research is an international, peer-reviewed open access journal focusing on cancer research and the optimal use of preventative and integrated treatment interventions to achieve improved outcomes, enhanced survival and quality of life for the cancer patient.
The manuscript management system is completely online and includes a very quick and fair peer-review system, which is all easy to use. Visit http://www.dovepress.com/testimonials.php to read real quotes from published authors. 